\title{
Spin-dependent polaron recombination in conjugated polymers
}

Zhen Sun and Sven Stafström

\section{Linköping University Post Print}

N.B.: When citing this work, cite the original article.

Original Publication:

Zhen Sun and Sven Stafström, Spin-dependent polaron recombination in conjugated polymers, 2012, Journal of Chemical Physics, (136), 24, 244901.

http://dx.doi.org/10.1063/1.4729483

Copyright: American Institute of Physics (AIP) http://www.aip.org/

Postprint available at: Linköping University Electronic Press http://urn.kb.se/resolve?urn=urn:nbn:se:liu:diva-79693 


\title{
Spin-dependent polaron recombination in conjugated polymers
}

\author{
Zhen Sun ${ }^{\text {a) }}$ and Sven Stafström ${ }^{\text {b) }}$ \\ Department of Physics, Chemistry, and Biology, Linköping University, SE-58183 Linköping, Sweden
}

(Received 26 March 2012; accepted 31 May 2012; published online 25 June 2012)

\begin{abstract}
We simulate the interchain polaron recombination process in conjugated polymer systems using a nonadiabatic molecular dynamics method, which allows for the coupled evolution of the nuclear degrees of freedom and multiconfigurational electronic wavefunctions. Within the method, the appropriate spin symmetry of the electronic wavefunction is taken into account, thus allowing us to distinguish between singlet and triplet excited states. It is found that the incident polarons can form an exciton, form a bound interchain polaron pair, or pass each other, depending on the interchain interaction strength and the strength of an external electric field. Most importantly, we found that the formation of singlet excitons is considerably easier than triplet excitons. This shows that in real organic light emitting devices, the electroluminescence quantum efficiency can exceed the statistical limitation value of 25\%, in agreement with experiments. () 2012 American Institute of Physics. [http://dx.doi.org/10.1063/1.4729483]
\end{abstract}

\section{INTRODUCTION}

Polaron recombination is the key step behind the mechanism of electroluminescence in organic light emitting devices (OLEDs). When two polarons with opposite charges overlap in space, they can recombine to form emissive singlet exciton (SE) or non-emissive triplet exciton (TE). Assuming that the polaron recombination process is spin-independent, simple statistical considerations give a ratio of singlet to the triplet of $1: 3$, which means that the maximum quantum efficiency of fluorescence-based OLEDs would be limited to $25 \%$. Nonetheless, a large number of observations of much higher quantum efficiencies have been reported in OLEDs made from conjugated polymers, including values as high as $83 \% .^{1-6}$ A considerable amount of theoretical work focused on understanding the mechanisms underlying exciton formation in conjugated polymers has also been reported..$^{7-11}$ So far, however, a commonly agreed theoretical picture is not available (for a review, see Ref. 12). On the other hand, SE yield above $25 \%$ in conjugated polymers continues to be debated. ${ }^{13-16}$

Recently, An et al. ${ }^{17}$ used the simple Su-SchriefferHeeger (SSH) Hamiltonian to simulate the polaron recombination process in conjugated polymers. Since the explicit electron-electron interactions are ignored in the SSH Hamiltonian, singlet and triplet excited states are not distinguishable. Li et al. ${ }^{18}$ and Lei et al. ${ }^{19}$ simulated the same process by adding Hubbard type electron-electron interactions to the SSH Hamiltonian. Even so, spin contamination could not be avoided in this approach because the unrestricted HartreeFock approximation was used to deal with the electronelectron interactions.

Very recently, Miranda et al. ${ }^{20,21}$ developed a multiconfigurational time-dependent Hartree-Fock (MCTDHF) method for the approximate solution of the time-dependent

\footnotetext{
a)Electronic mail: zhesu@ifm.liu.se.

b) Electronic mail: svens@ifm.liu.se.
}

Schrödinger equation for large systems of interacting electrons. The method was designed to take into account the appropriate spin symmetry of the electronic wavefunction by retaining the smallest possible number of configurations that catches the essential features of the electronic wavefunctions, which establishes a compromise between efficiency and accuracy and allows us to distinguish between singlet and triplet excited states. In this paper, we adopt the formalism developed by Miranda et al., briefly described in Sec. II, to investigate the recombination process between a negative and a positive polaron in a coupled two-chain system in the presence of an external electric field. We focus on the differences between the singlet and triplet recombination processes as a function of the interchain strength and as a function of the strength of the external electric field.

\section{MODEL AND METHOD}

We consider a system composed by two coupled polymer chains. Each chain has 180 sites: the sites in chain 1 are labelled 1-180, while the sites in chain 2 are labelled 181-360, and the number on the leftmost atom of the second chain is 181. The two chains are superimposed in a co-facial configuration. The overall Hamiltonian for this composite two-chain system is given by

$$
\hat{H}_{t o t}=\hat{H}_{l a t t}+\hat{H}_{\text {elec }} .
$$

The first term in Eq. (1) is the Hamiltonian of the lattice backbone, which is treated classically: ${ }^{22}$

$$
\hat{H}_{\text {latt }}=\frac{K}{2} \sum_{i}\left(u_{i+1}-u_{i}\right)^{2}+\frac{M}{2} \sum_{i} \dot{u}_{i}^{2}
$$

where $K$ is the elastic constant of a $\sigma$ bond, $M$ the mass of a $\mathrm{CH}$ group, and $u_{i}$ the lattice displacement of the $i$ th site from its equidistant position. 
The second term in Eq. (1) is the Hamiltonian of the electronic part of the system,

$$
\hat{H}_{e l e c}=\hat{H}_{e l}+\hat{H}_{e e}+\hat{H}_{E} .
$$

In the above expression, the first contribution denotes the electron transfer integral, expressed as ${ }^{22}$

$$
\hat{H}_{e l}=-\sum_{i, s} t_{i j}\left(\hat{c}_{i, s}^{\dagger} \hat{c}_{j, s}+\hat{c}_{j, s}^{\dagger} \hat{c}_{i, s}\right),
$$

where $\hat{c}_{i, s}^{\dagger}$ and $\hat{c}_{i, s}$ are the creation and annihilation operators, respectively, for an electron on site $i$, and

$$
t_{i, j}= \begin{cases}t_{0}-\alpha\left(u_{i}-u_{j}\right)+(-1)^{i} t_{e} & \text { intrachain hopping } \\ & \text { with } j=i \pm 1, \\ t_{\perp} \text { or } t_{d} & \text { interchain hopping } \\ & \text { (see below) }\end{cases}
$$

with $t_{0}$ denoting the hopping integral of $\pi$ electrons for zero lattice displacements, $\alpha$ the electron-phonon coupling constant, and $t_{e}$ the symmetry-breaking term, which is introduced to lift the ground state degeneracy. $t_{\perp}$ is the orthogonal hopping integral, i.e., the hopping between a site on one chain and a nearest neighbor site on an adjacent chain, and $t_{d}$ the diagonal hopping integral, i.e., the hopping between next nearest neighbor site on adjacent chains. $t_{\perp}$ and $t_{d}$ are functions of the interchain distance $d$, using ${ }^{23}$

$$
\begin{gathered}
t_{\perp}=\frac{t_{0}}{10} \exp \left(1-\frac{d}{5}\right), \\
t_{d}=\frac{t_{0}}{10} \exp \left(1-\frac{\sqrt{d^{2}+r_{0}^{2}}}{5}\right),
\end{gathered}
$$

where $r_{0}$ is the average bond length.

The second contribution in Eq. (3) denotes long-range electron-electron interactions and can be written as

$$
\begin{aligned}
\hat{H}_{e e}= & U \sum_{i, s}\left(\hat{c}_{i, s}^{\dagger} \hat{c}_{i, s}-\frac{1}{2}\right)\left(\hat{c}_{i,-s}^{\dagger} \hat{c}_{i,-s}-\frac{1}{2}\right) \\
& +\frac{1}{2} \sum_{i, j, s, s^{\prime}} V_{i j}\left(\hat{c}_{i, s}^{\dagger} \hat{c}_{i, s}-\frac{1}{2}\right)\left(\hat{c}_{j, s^{\prime}}^{\dagger} \hat{c}_{j, s^{\prime}}-\frac{1}{2}\right),
\end{aligned}
$$

where $U$ and $V_{i j}$ denote the on-site and intersite Coulomb interactions, respectively. For $V_{i j}$, we used the Ohno potential defined as

$$
V_{i j}=\frac{U}{\sqrt{1+\left(\beta r_{i j} / r_{0}\right)^{2}}},
$$

where $\beta$ the screening factor and $r_{i j}$ denotes the distance between site $i$ and $j$.

The last contribution in Eq. (3) represents the external electric field and can then be written in the form

$$
\hat{H}_{E}=|e| E(t) \sum_{i, s}\left[(i-1) r_{0}+u_{i}\right]\left(\hat{c}_{i, s}^{\dagger} \hat{c}_{i, s}-\frac{1}{2}\right),
$$

where $e$ denotes the electronic charge. In our simulations, the field $E(t)$ is constant after a smooth turn on.

The set of parameters used in the calculations are chosen so as to model cis-polyacetylene. Miranda et $a l^{21}$ developed a set of parameters for cis-polyacetylene in order to solve the inconformity of parameters when Coulomb interactions are included in the Hamiltonian. Thus, in this paper, we adopt the parameters developed by them: $t_{0}=2.1 \mathrm{eV}, \alpha=3.2 \mathrm{eV} / \AA$, $t_{e}=0.05 \mathrm{eV}, K=21.0 \mathrm{eV} / \AA^{2}, M=1349.14 \mathrm{eVfs}^{2} / \AA^{2}, r_{0}$ $=1.22 \AA, U=4.1 \mathrm{eV}$, and $\beta=3.4$.

Within the nonadiabatic molecular dynamics method, the electrons are treated quantum-mechanically and evolve according to the time-dependent Schrödinger equation,

$$
i \hbar|\dot{\Psi}\rangle=\hat{H}_{\text {elec }}|\Psi\rangle .
$$

The atoms, on the other hand, are considered as classical particles, governed by Newton equations, and move under the effects of nuclear and electronic contributions,

$$
M \ddot{u}_{i}=-\nabla_{i}\left\langle\Psi\left|\hat{H}_{\text {latt }}\right| \Psi\right\rangle-\nabla_{i}\left\langle\Psi\left|\hat{H}_{\text {elec }}\right| \Psi\right\rangle .
$$

The solution of the full many-body problem given in Eq. (11) is generally not possible and approximate methods are necessary. As we have mentioned above, we use a MCTDHF method introduced in Refs. 20 and 21 to solve this equation. Within this method, the appropriate spin symmetry of the electronic wavefunction is taken into account, thus allowing us to distinguish between singlet and triplet excited states. Equations (11) and (12), which govern the evolution of the system, may be numerically integrated using the method, which was first introduced by Ono and Terai ${ }^{24}$ and has been used in our previous papers. ${ }^{25-28}$ It should be noted that the MCTDHF method allows for a manifold of states to be involved in the dynamic process. In particular, in between the initial state of two separated polarons and the final SE or TE state, we observe several intermediate states, the most important one being the state of a bound polaron pair (see Sec. III below).

The starting geometry is a negative polaron in one chain located at site 50 and a positive polaron in the other chain located at site 310 . This geometry is obtained by minimizing the total energy of one chain with an extra electron (without the presence of the electric field) and the same for the other chain, but with an extra hole. Then, we join the two chains by gradually adding the interchain interactions. Also, the external electric field is turned on adiabatically in order to avoid extrinsic effects to the dynamics of the system. The negative and positive polarons then move towards each other under the influence of the electric field and depending on their velocity, which in turn depends on the strength of the electric field, they will start to overlap at 200-250 fs after the field was turned on.

In the result section below, we use the staggered order parameter $r_{i}(t)$ and the mean charge density $\bar{\rho}_{i}(t)$ to analyze and display the lattice and charge density evolution,

$$
\begin{gathered}
r_{i}(t)=\frac{(-1)^{i}}{4}\left[u_{i-1}(t)-2 u_{i}(t)+u_{i+1}(t)\right], \\
\bar{\rho}_{i}(t)=\frac{1}{4}\left[\rho_{i-1, i-1}(t)+2 \rho_{i, i}(t)+\rho_{i+1, i+1}(t)\right],
\end{gathered}
$$

where the charge density $\rho_{i, j}(t)=\sum_{s}\left\langle\hat{c}_{i, s}^{\dagger} \hat{c}_{j, s}\right\rangle$. 


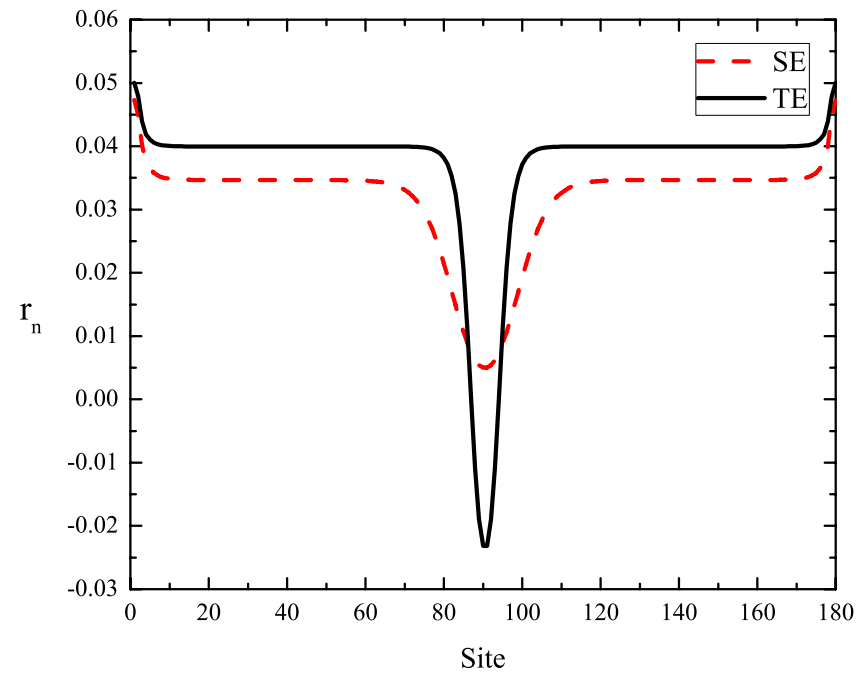

FIG. 1. Optimized staggered order parameter for a SE and a TE in single polymer chain.

\section{RESULTS AND DISCUSSIONS}

Figure 1 shows the geometries of $\mathrm{SE}$ and TE in a single polymer chain, which are obtained by minimizing the total energy of the first excited state of a single polymer chain. The most distinct difference between the two excited states is that the TE is more localized than the SE. This is in agreement with other theoretical predictions and experimental observations. ${ }^{29,30}$ Moreover, the distortion at the center of the SE is shallower than the TE, and the dimerization is also different between the two states. In addition, we note that the value for the exciton singlet-triplet splitting is about $0.7 \mathrm{eV}$, which is roughly equal to the experimentally observed value in a poly-p-phenylene-vinylene derivative. ${ }^{5}$ These different properties of SE and TE indicate that the polaron recombination processes into a SE or a TE also may be different.

We then turn to the coupled two-chain system. A first set of simulations was carried out for a number of different interchain distances with fixed electric field $0.5 \mathrm{mV} / \AA ̊$. In Fig. 2, we present the temporal evolution of the mean charge density $\bar{\rho}_{i}(t)$ for both singlet and triplet spin states. Panels a1, a2, and a3 correspond to singlet state recombination, while panels b1, $\mathrm{b} 2$, and $\mathrm{b} 3$ to triplet state recombination. The top, middle, and bottom panels correspond to interchain distances $10 \AA, 15 \AA$, and $20 \AA$, respectively. At first, we focus on panels a1 and b1. We can see that there are no obvious differences between singlet and triplet polaron recombination processes. Under the influence of the electric field, the two polarons move towards each other and meet after about 250 fs. Then they bind together forming a polaron pair. ${ }^{31}$ About $180 \mathrm{fs}$ after their first encounter, the charges of the two polarons cancel each other. It is not difficult to conjecture that the charge cancellation is due to the formation of exciton. In order to verify our conjecture, we draw the evolution of the staggered order parameter $r_{i}(t)$ with the same interchain distance and electric field in Fig. 3. From Fig. 3, we find a large lattice distortion formed after recombination. By comparing with panels a1 and b1 of Fig. 2, this distortion is neutral and localized to a single chain and agrees very well with the distortion presented in Fig. 1. Together with the evolution of the intra-gap levels and their
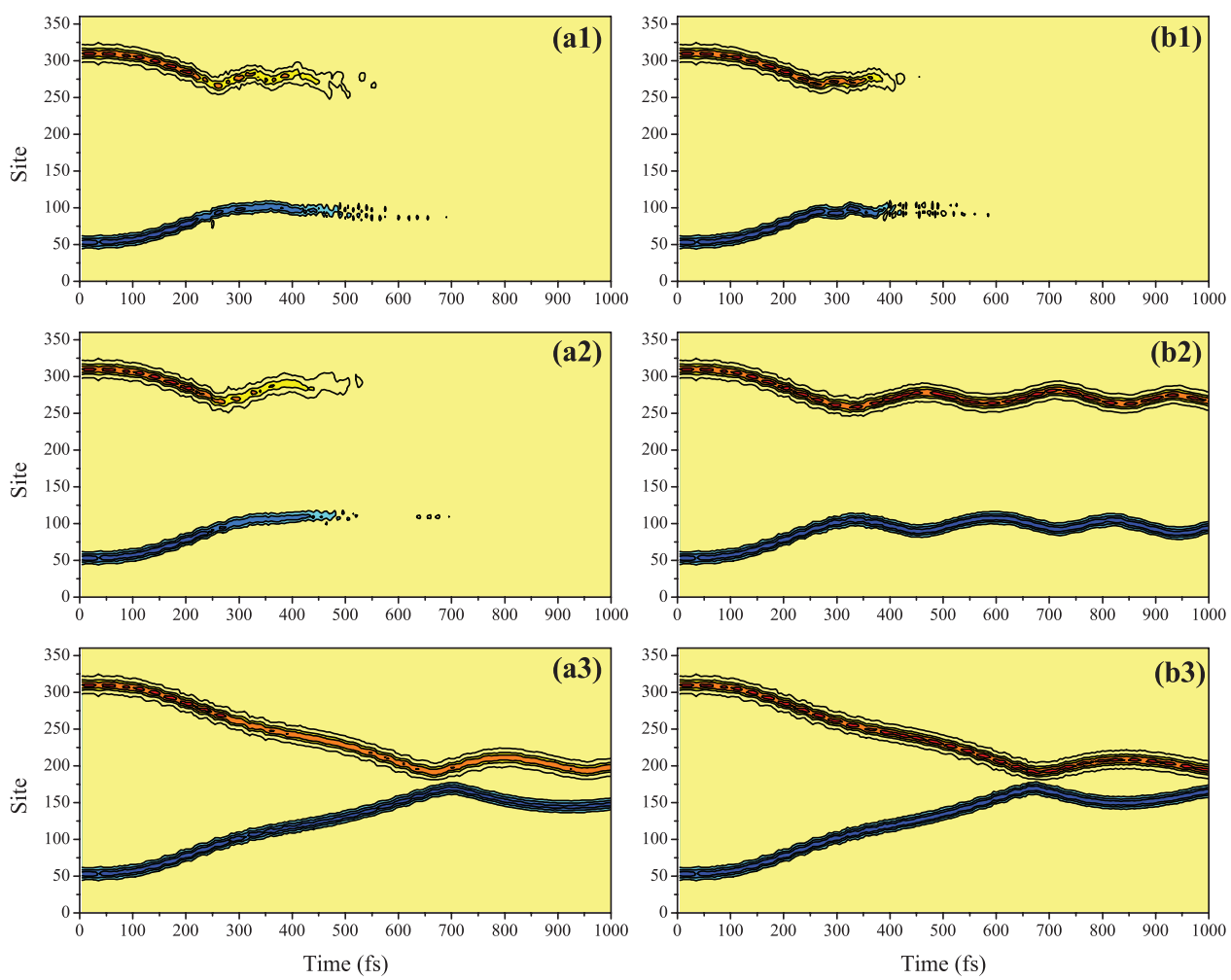

FIG. 2. Time dependence of $\bar{\rho}_{i}$ for polaron recombination in the singlet [(a1), (a2), and (a3)] and triplet spin [(b1), (b2), and (b3)] states, respectively. Three different interchain distance are considered: $\mathrm{d}=10 \AA$ (top panel), $\mathrm{d}=15 \AA$ (middle panel), and $\mathrm{d}=20 \AA$ (bottom panel), $\mathrm{E}=0.5 \mathrm{mV} / \AA$. In panel (a1), (a2), and (b1), we can see that charge recombination has occurred. After that, isolated charges are not detected anymore. 

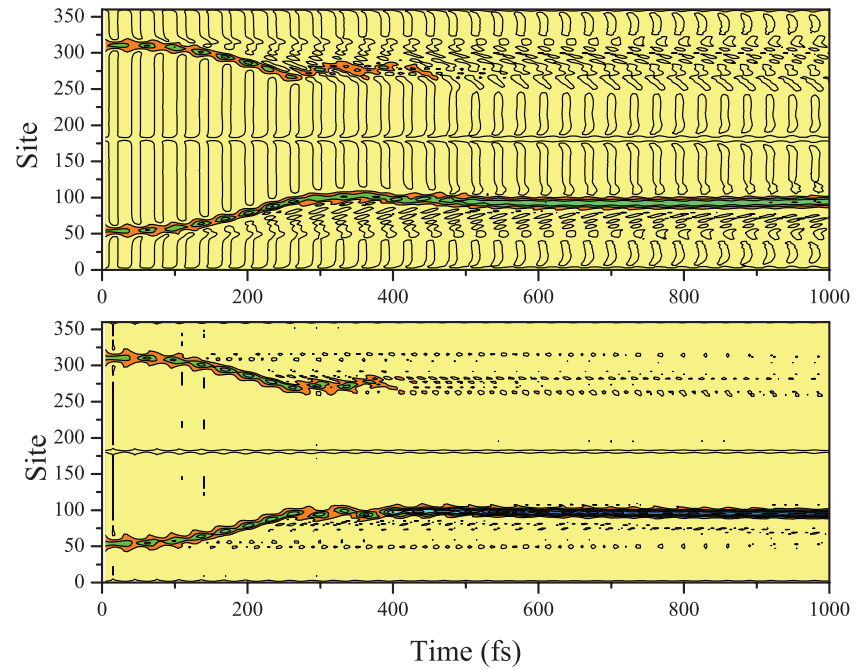

FIG. 3. Time dependence of $r_{i}$ for polaron recombination in the singlet (top panel) and triplet spin (bottom panel) states, respectively, and with $\mathrm{d}=10 \AA$ and $\mathrm{E}=0.5 \mathrm{mV} / \AA$.

occupation numbers (not shown in this paper), we conclude that an exciton is formed in both the singlet and triplet cases.

However, if we increase the interchain distance, very interesting differences appear between the singlet and triplet excited states. For the singlet state, the two polarons recombine into an exciton for interchain distance up to and including $\mathrm{d}=18 \AA$ A, i.e., very weakly interacting polymer chains. For a slightly greater interchain distance, the interchain interactions become too weak for the the two polarons to overlap sufficiently. In this situation, they pass each other as can be seen from panel a3 in Fig. 2. In contrast, in the triplet state, the two polarons cannot recombine into an exciton for interchain distance $\mathrm{d}=14 \AA$, i.e., in order for the triplet exciton to form the chains have to interact much stronger than in the singlet case. Instead of recombining, the two polarons bound together forming a stable polaron pair (see Fig. 2, panel b2). This occurs for interchain distances between $14 \AA$ and $17 \AA$. For $\mathrm{d}>17 \AA$, the two polarons pass each other as is shown in panel b3 in Fig. 2. This clearly shows that SEs are easier formed than TEs in case of large interchain distances.

As can be seen from panels a 2 and b2 of Fig. 2, a stable polaron pair is only formed in the case of the triplet state and not for the singlet state. The polaron pair formation is due to the large energy difference between the incident polarons and the TE, which in the case of weakly interacting chains, prevents direct recombination into the TE state. In the case of the SE, however, the larger exchange energy (energy difference between SE and TE) results in a lower energy difference between the incident polarons and the SE, which facilitates the direct recombination into SE state. ${ }^{8,9}$

Simulations were also carried out for a number of different electric field strengths. It was found that the electric field also affected the polaron recombination processes, but in different ways for singlet and triplet states. In the following, we fixed the interchain distance to $d=10 \AA$ and studied the recombination process for different strengths of the electric field. For the singlet state, our simulations showed that the two polarons recombined into an exciton for E up
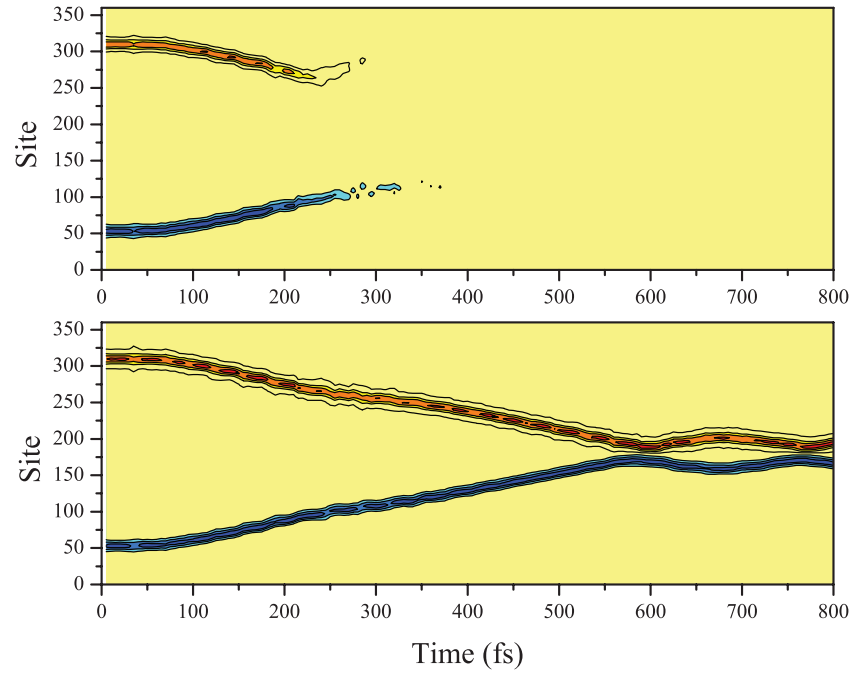

FIG. 4. Time dependence of $\bar{\rho}_{i}$ for polaron recombination in the singlet (top panel) and triplet spin (bottom panel) states, respectively, and with $\mathrm{d}=10 \AA$ and $\mathrm{E}=1.5 \mathrm{mV} / \AA$.

to $2.6 \mathrm{mV} / \AA$. For a slightly higher field strength, the two polarons passed each other without interacting. In contrast, for the triplet state, the two polarons only recombined into an exciton for field strengths below $\mathrm{E}=1.5 \mathrm{mV} / \AA$, and passed each other for field strengths above this value. The difference in the behavior between the two spin states is illustrated in Fig. 4, which shows the temporal evolution of the mean charge density $\bar{\rho}_{i}(t)$ for both singlet (top panel) and triplet (bottom panel) states with $\mathrm{d}=10 \AA$ and $\mathrm{E}=1.5 \mathrm{mV} / \AA$. Similar to the simulations for varying interchain interactions, this shows that the singlet state has a considerably stronger tendency to form an exciton.

\section{CONCLUSIONS}

Based on the results presented above, it is possible to understand why the electroluminescence quantum efficiency of the OLED can exceed the statistical limitation value of $25 \%$. In a real OLED, the distances between polymer chains are diverse, and the local electric field strengths are different due to the random orientations of the polymer chains. When two polarons approach each other, a larger fraction of them will recombine into an exciton state if they are in a singlet spin state than if the spin state is a triplet. Consequently, a larger fraction of the polarons in the triplet state will simply pass each other. These polarons will, at a later stage, have the chance to form singlet states that can contribute to the electroluminescence.

In summary, we have simulated the interchain polaron recombination process both for singlet and triplet spin states. We found that the two polarons can recombine into an exciton, bound together forming a polaron pair or pass each other depending on the interchain distance and the electric field strength. Most importantly, we found that the SEs are always easier to be formed than the TEs. This indicates that in a real OLEDs, the electroluminescence quantum efficiency can exceed the statistical limitation value of $25 \%$, in agreement with the experiments. 


\section{ACKNOWLEDGMENTS}

The authors would like to thank Patrick Norman and Bo Durbeej for valuable discussions. Financial support from the Swedish Research Council and the Swedish Energy Agency is gratefully acknowledged.

${ }^{1}$ Y. Cao, I. D. Parker, G. Yu, C. Zhang, and A. J. Heeger, Nature (London) 397, 414 (1999).

${ }^{2}$ P. K. H. Ho, J. S. Kim, J. H. Burroughes, H. Becker, S. F. Y. Li, T. M. Brown, F. Cacialli, and R. H. Friend, Nature (London) 404, 481 (2000).

${ }^{3}$ M. Wohlgenannt, K. Tandon, S. Mazumdar, S. Ramasesha, and Z. V. Vardeny, Nature (London) 409, 494 (2001).

${ }^{4}$ M. Wohlgenannt, X. M. Jiang, Z. V. Vardeny, and R. A. J. Janssen, Phys. Rev. Lett. 88, 197401 (2002).

${ }^{5}$ J. S. Wilson, A. S. Dhoot, A. J. A. B. Seeley, M. S. Khan, A. Köhler, and R. H. Friend, Nature (London) 413, 828 (2001).

${ }^{6}$ A. S. Dhoot, D. S. Ginger, D. Beljonne, Z. Shuai, and N. C. Greenham, Chem. Phys. Lett. 360, 195 (2002).

${ }^{7}$ Z. Shuai, D. Beljonne, R. J. Silbey, and J. L. Brédas, Phys. Rev. Lett. 84, 131 (2000).

${ }^{8}$ K. Tandon, S. Ramasesha, and S. Mazumdar, Phys. Rev. B 67, 045109 (2003).

${ }^{9}$ M. N. Kobrak and E. R. Bittner, Phys. Rev. B 62, 11473 (2000).

${ }^{10}$ S. Karabunarliev and E. R. Bittner, Phys. Rev. Lett. 90, 057402 (2003).

${ }^{11}$ T. M. Hong and H. F. Meng, Phys. Rev. B 63, 075206 (2001).

${ }^{12}$ M. Wohlgenannt and Z. V. Vardeny, J. Phys.: Condens. Matter 15, R83 (2003).

${ }^{13}$ M. Segal, M. A. Baldo, R. J. Holmes, S. R. Forrest, and Z. G. Soos, Phys. Rev. B 68, 075211 (2003).

${ }^{14}$ M. K. Lee, M. Segal, Z. G. Soos, J. Shinar, and M. A. Baldo, Phys. Rev. Lett. 94, 137403 (2005).
${ }^{15}$ M. Segal, M. A. Baldo, M. K. Lee, J. Shinar, and Z. G. Soos, Phys. Rev. B 71, 245201 (2005).

${ }^{16}$ M. Reufer, M. J. Walter, P. G. Lagoudakis, A. B. Hummel, J. S. Kolb, H. G. Roskos, U. Scherf, and J. M. Lupton, Nature Mater. 4, 340 (2005).

${ }^{17}$ Z. An, B. Di, H. Zhao, and C. Q. Wu, Eur. Phys. J. B 63, 71 (2008).

${ }^{18}$ Y. Li, K. Gao, Z. Sun, S. Yin, D. S. Liu, and S. J. Xie, Phys. Rev. B 78, 014304 (2008).

${ }^{19}$ J. Lei, Z. Sun, Y. B. Zhang, and S. J. Xie, Org. Electron. 10, 1489 (2009).

${ }^{20}$ R. P. Miranda, A. J. Fisher, L. Stella, and A. P. Horsfield, J. Chem. Phys. 134, 244101 (2011).

${ }^{21}$ R. P. Miranda, A. J. Fisher, L. Stella, and A. P. Horsfield, J. Chem. Phys. 134, 244102 (2011).

${ }^{22}$ A. J. Heeger, S. Kivelson, J. R. Schrieffer, and W. P. Su, Rev. Mod. Phys. 60, 781 (1988)

${ }^{23}$ E. J. McEniry, Y. Wang, D. Dundas, T. N. Todorov, L. Stella, R. P. Miranda, A. J. Fisher, A. P. Horsfield, C. P. Race, D. R. Mason, W. M. C. Foulkes, and A. P. Sutton, Eur. Phys. J. B 77, 305 (2010).

${ }^{24}$ Y. Ono and A. Terai, J. Phys. Soc. Jpn. 59, 2893 (1990).

${ }^{25}$ Z. Sun and S. Stafström, J. Chem. Phys. 135, 074902 (2011).

${ }^{26}$ Z. Sun, D. S. Liu, S. Stafström, and Z. An, J. Chem. Phys. 134, 044906 (2011).

${ }^{27}$ Z. Sun, Y. Li, K. Gao, D. S. Liu, Z. An, and S. J. Xie, Org. Electron. 11, 279 (2010).

${ }^{28}$ Z. Sun, Y. Li, S. J. Xie, Z. An, and D. S. Liu, Phys. Rev. B 79, 201310(R) (2009).

${ }^{29}$ D. Beljonne, Z. Shuai, R. H. Friend, and J. L. Brédas, J. Chem. Phys. 102, 2042 (1995).

${ }^{30}$ A. Köler, J. S. Wilson, R. H. Friend, M. K. Al-Suti, M. S. Khan, A. Gerhard, and H. Bäsler, J. Chem. Phys. 116, 9457 (2002).

${ }^{31}$ E. L. Frankevich, A. A. Lymarev, I. Sokolik, F. E. Karasz, S. Blumstengel, R. H. Baughman, and H. H. Hörhold, Phys. Rev. B 46, 9320 (1992). 